\title{
ANALYTIC PROPERTIES OF THE LONGITUDINAL BEAM TRANSFER FUNCTION
}

\author{
N. Towne*, National Synchrotron Light Source \\ Brookhaven National Laboratory, Bldg. 725B, Upton, NY 11973-5000 ${ }^{\dagger}$
}

\begin{abstract}
The frequency dependence of the longitudinal beam transfer function (BTF) in a storage ring, when expressed in a basis of azimuthal harmonics of the line density, is the Fourier transform of a causal function that depends on the radio-frequency potential well in which the bunch moves. The effect of all synchrotron harmonics are included in this function, which is derived from Krinsky and Wang's expression for the BTF expressed in the same basis (S. Krinsky and J.-M. Wang, Part. Accel. 17, 109-139 (1985)). Analytic properties of the terms of the BTF expressed in a series of synchrotron harmonics, which are approximately Shaposhnikova's BTF matrix elements (E. Shaposhnikova, CERN Report No. SL-94-19-RF (1994)), are studied through the large-argument asymptotics of corresponding causal functions.
\end{abstract}

\section{INTRODUCTION}

Krinsky and Wang (K \& W) developed an expression for the longitudinal beam transfer function (BTF) $G_{m n}(\Omega)$ in a storage ring, which is the frequency-domain response of a bunch to a total-voltage excitation obtained by an inversion procedure applied to the Vlasov equation [1]. Their expression is valid for radio-frequency (rf) potentials with monotonic synchrotron frequencies and is expressed in a basis of azimuthal harmonics. It also serves as a basis for derivation of part of Shaposhnikova's explicit formulation of the BTF and her BTF matrix elements $M_{\mu}^{m n}(\Omega)$ [2], the latter obtained through the use of Lebedev's synchrotronharmonic expansion [3].

Although Krinsky and Wang's expression has a singular denominator that is difficult to work with directly, it permits insertion of a series expansion for a singular denominator that results in an expression for $G_{m n}(\Omega)\left(G_{m n}(\Omega)\right.$ relates excitations near the $n$th revolution harmonic to the beam's response near the $m$ th revolution harmonic) with frequency dependence that is the Fourier transform of a causal function $h_{m n}(t)$ determined by the single-particle dynamics. A similar procedure can be applied to $M_{\mu}^{m n}$. This function is readily calculated and provides a numerical method, when inserted into dispersion relations, by which coherent frequencies including all synchrotron harmonics may be calculated in one step (Sec. 2).

What is also interesting is that, since the analytic proper-

\footnotetext{
*Email: towne@bnl.gov

$\dagger$ Work performed under the auspices of the U.S. Department of Energy, under contract DE-AC02-76CH00016.
}

ties of the Fourier transform of a function $f(t)$ are closely linked to the $t \rightarrow \infty$ asymptotics of $f$, an assessment of the analytic properties of $G_{m n}$ through the asymptotics of $h_{m n}$ may be made, at least numerically. Stronger statements are developed for the functions $M_{\mu}^{m n}(\Omega)$ where the expression for $\tilde{M}_{\mu}^{m n}(t)$ is also an integral transform but now with an exponential kernel. If a suitable change of variable of integration can be made, the integral transform is a Fourier transform and the $t \rightarrow \infty$ asymptotics is closely related to the differentiability properties of the object of the integral transform representing $\tilde{M}_{\mu}^{m n}(t)$. A sufficient condition under which the $t \rightarrow \infty$ asymptotics of $\tilde{M}_{\mu}^{m n}(t)$ is exponentially decreasing, requiring $M_{\mu}^{m n}(\Omega)$ to be regular for $\Omega$ on the real axis, is derived (Sec. 3).

\section{FOURIER TRANSFORM EXPRESSION FOR THE BEAM TRANSFER FUNCTION}

First take a moment to become familiar with the notation used here. The phase-space variables $J$ and $\theta$ are the action-angle canonical variables with respect to the radio-frequency (rf) Hamiltonian. The variable $\phi$ is the co-moving coordinate related to the azimuthal angle $\psi$ through $\psi=\omega_{0} t+\phi$, where $\omega_{0}$ is the revolution frequency. There is a variable $p$ canonically conjugate to $\phi$. The rf Hamiltonian is $H=H(J)$ and the rf potential is $U_{\mathrm{rf}}=U_{\mathrm{rf}}(\phi)$. In terms of $\phi$ and $p, H(J)=H(\phi, p)=$ $p^{2} / 2+U_{\mathrm{rf}}(\phi)$. The synchrotron frequency $\omega_{s}$ is a function of $J$ and $T_{s}(J)=1 / \omega_{s}(J)$. The static particle distribution in phase space is $\Psi_{0}(J) \propto e^{-H / \sigma^{2}}$, where $\sigma=\alpha \omega_{0} \sigma_{\epsilon}$, $\alpha$ is the momentum compaction, and $\sigma_{\epsilon}$ is the fractional energy spread of the ring. Krinsky and Wang's BTF is expressed as $F_{m n}=-G_{m n} / \kappa$ in their notation, where $\kappa=e \alpha \omega_{0}^{3} / 4 \pi^{2} E_{0}$ and $E_{0}$ is the nominal particle energy.

The $\mathrm{K} \& \mathrm{~W}$ expression for $F_{m n}(\Omega)$ is an integral,

$$
\begin{aligned}
F_{m n}(\Omega)= & \frac{1}{i n} \int_{0}^{\infty} d J \frac{\Psi_{0}^{\prime}(J)}{\omega_{s}(J)\left(1-e^{i \Omega T_{s}(J)}\right)} \\
& \times \int_{-2 \pi}^{0} d \theta^{\prime} e^{-i \Omega T_{s} \theta^{\prime} / 2 \pi} \partial_{\theta^{\prime}} g_{m n}\left(J, \theta^{\prime}\right)(1)
\end{aligned}
$$

where

$$
g_{m n}\left(J, \theta^{\prime}\right)=\int_{2 \pi} d \theta e^{-i\left(m \phi(J, \theta)-n \phi\left(J, \theta+\theta^{\prime}\right)\right)}
$$

The series $1 /\left(1-e^{i \Omega T_{s}(J)}\right)=\sum_{l=0}^{\infty} e^{i \Omega l T_{s}}$, which is valid for $\Omega$ with positive imaginary part, is inserted into Eq. (1). 
The terms of the series are then combined with the integral with respect to $\theta$ to extend the lower limit of integration to $-\infty$, the variable of integration $\theta^{\prime}$ is changed to $\tau^{\prime}=$ $\theta^{\prime} / \omega_{s}(J)$, and the derivatives of $\Psi_{0}$ and $g_{m n}$ are combined into the scalar Poisson bracket $\left\{g_{m n}, \Psi_{0}\right\}$.

$$
F_{m n}(\Omega)=\frac{1}{i n} \int_{0}^{\infty} d J \int_{-\infty}^{0} d \tau^{\prime} e^{-i \Omega \tau^{\prime}}\left\{g_{m n}, \Psi_{0}\right\}
$$

Even though $\tau^{\prime}$ was originally defined as dependent upon $J$, the semi-infinite limits of integration permit this to be ignored and the order of integration to be interchanged. Changing variable of integration from $\tau^{\prime}$ to $\tau=-\tau^{\prime}$, we have the one-sided Fourier transform

$$
G_{m n}(\Omega)=-\kappa F_{m n}(\Omega)=\frac{1}{i n} \int_{0}^{\infty} d \tau e^{i \Omega \tau} h_{m n}(\tau)
$$

where

$$
h_{m n}(\tau)=-\kappa \int_{0}^{\infty} d J\left\{g_{m n}, \Psi_{0}\right\}
$$

and the $\theta$ dependence of the Poisson brackets is through $\theta=\omega_{s}(J) \tau^{\prime}=-\omega_{s}(J) \tau$.

A simpler approximate expression for $h_{m n}$ is obtained by inserting the familiar Bessel-function substitution in place of the Fourier coefficients of the exponential

$$
\begin{aligned}
e^{i n \phi(J, \theta)} & =\sum_{\mu=-\infty}^{\infty} F_{\mu}(n, J) e^{i \mu \theta} \\
& \simeq \sum_{\mu=-\infty}^{\infty} i^{\mu} J_{\mu}(n r(J)) e^{i \mu \theta}
\end{aligned}
$$

where $r=r(J)$ is the radial variable that is the maximum value of $\phi$ on the trajectory with action variable $J$ and $J_{\mu}$ is the Bessel function of the first kind. Eq. (7) is then substituted into Eq. (2). A Bessel-function identity is employed to get

$$
g_{m n}(J, \theta) \simeq 2 \pi J_{0}\left(r(J) R_{m n}(\theta)\right)
$$

where $R_{m n}(\theta)=\sqrt{m^{2}+n^{2}-2 m n \cos \theta}$. Inserted into Eq. (5), $h_{m n}$ is much more easily computed using Eq. (8) than when using the exact Eq. (2).

Numerical evidence that $G_{m n}(\Omega)$ is analytic was found using the approximate Eq. (8) and Eq. (5). As was noted earlier, the $\tau \rightarrow \infty$ asymptotic behavior of $h_{m n}(\tau)$ determines the region of the complex plane over which $G_{m n}(\Omega)$ is regular. In particular, $G_{m n}$ is regular on the real axis if $h_{m n}$ is bounded by a decaying exponential with increasing $\tau$. In the case of the model $U_{\mathrm{rf}}(\phi)=b \phi^{4} / 4$ potential approximating the potential used to stretch bunches, numerical calculation shows that $h_{m n}(\tau)$ of Eq. (5) with the approximate Eq. (8) inserted decreases in magnitude at least as fast as exponentially to $\Delta \omega_{s} \tau=80$ where the dimensionless integral has magnitude of order $10^{-35}$ $\left(\Delta \omega_{s}=0.849\left(4 b \alpha^{2} \omega_{0}^{2} \sigma_{\epsilon}^{2}\right)^{1 / 4}\right)$. Consequently, $G_{m n}$, as defined with the approximate form Eq. (8), is likely regular on a half plane of the $\Omega$ plane containing the real axis. The numerical data also suggest that the decay is faster than exponential, implying that $G_{m n}$ is an entire function of $\Omega$. Contrast this behavior to the general quartic potential $U_{\text {rf }}(\phi)=\omega_{s 0}^{2} \phi^{2} / 2+b \phi^{4} / 4$, where $\omega_{s 0}$ and $b$ are nonzero constants; in this case the oscillatory $h_{m n}(\tau)$ decreases as $\tau^{-2}$. Consequently, $G_{m n}(\Omega)$ has singularities on the real axis in this case.

\section{SHAPOSHNIKOVA'S BTF MATRIX ELEMENTS}

Shaposhnikova developed a representation of $G_{m n}$ in a series of matrix elements $M_{\mu}^{m n}(\Omega)$ in synchrotron harmonics [2] that is closely related to Lebedev's dispersion relation [3]. It may be derived by first inserting Eq. (6) into Eq. (2) with the result

$$
g_{m n}(J, \theta)=2 \pi \sum_{\mu=-\infty}^{\infty} F_{\mu}^{*}(m, J) F_{\mu}(n, J) e^{i \mu \theta}
$$

This series is inserted into Eq. (5), the order of integration and summation is interchanged, and individual terms extracted. The result is

$$
h_{m n}(\tau)=-\kappa \sum_{\mu=1}^{\infty} \tilde{M}_{\mu}^{m n}(\tau)
$$

where the terms are

$$
\begin{aligned}
\tilde{M}_{\mu}^{m n}(\tau)= & 2 \pi i \mu \int_{0}^{\infty} d J \Psi_{0}^{\prime}(J) \\
& \times \sum_{ \pm} \pm F_{ \pm \mu}^{*} F_{ \pm \mu} e^{\mp i \mu \omega_{s} \tau}
\end{aligned}
$$

The $m, n$, and $J$ dependence of $F_{\mu}$ and the $J$ dependence of $\omega_{s}$ are suppressed. Analogous to expressing $G_{m n}(\Omega)$ as the Fourier transform of the quantity $h_{m n}(\tau)$, we express $M_{\mu}^{m n}(\Omega)$ as the one-sided Fourier transform

$$
M_{\mu}^{m n}(\Omega)=\int_{0}^{\infty} d \tau e^{i \Omega \tau} \tilde{M}_{\mu}^{m n}(\tau)
$$

These functions are Shaposhnikova's $M_{\mu}^{m n}(\Omega)$ up to an $n$-dependent factor. The functions $\tilde{M}_{\mu}^{m n}(\tau)$ contain information about the analytic properties of $M_{\mu}^{m n}(\Omega)$ analogous to $h_{m n}(\tau)$ and $G_{m n}(\Omega)$. But now $\tilde{M}_{\mu}^{m n}(\tau)$ is expressed in terms of integral transforms with kernels $\exp \left( \pm i \mu \omega_{s}(J) \tau\right)$.

In general, the $J$ dependence of $\omega_{s}$ does not permit $\tilde{M}_{\mu}^{m n}(\tau)$ to decrease asymptotically fast enough as $\tau \rightarrow \infty$ for $M_{\mu}^{m n}(\Omega)$ to be regular on the real axis. But if $\omega_{s}(J)$ permits the $J$ variable of integration to be changed to $\omega_{s}$ in such a way that the domain of $\omega_{s}$ is the entire real axis and invoking symmetry properties, then Eq. (11) becomes a two-sided Fourier transform. If the integrand is regular in $\omega_{s}$ over this domain and the integrand decreases sufficiently quickly as $\omega_{s} \rightarrow \pm \infty$ ( $\Psi_{0}$ assures this), then $\tilde{M}_{\mu}^{m n}(\tau)$ is bounded by a decaying exponential in $\tau$ [4]. This ensures that $M_{\mu}^{m n}(\Omega)$ is regular for $\Omega$ on a half plane 
containing the real axis. So the problem is first, to see how $\omega_{s}$ can be an infinitely differentiable $\left(C^{\infty}\right)$ function on the phase space and, second, to identify sufficient conditions that ensure that the integrand of Eq. (11) is an analytic function of $\omega_{s}$ for $-\infty<\omega_{s}<\infty$.

It is useful to switch the radial variable from $J$ to $r$ since $r=r(\phi, p=0)=\phi$ is a $C^{\infty}$ function. Then consider the properties of $\omega_{s}(r)$. When $\omega_{s}(r)$ has a minimum $\omega_{s}(0)=\omega_{s 0}>0$, it is not possible for the domain of $r\left(\omega_{s}\right)$ to extend to zero, at least for real $\omega_{s}$. In this case, $\omega_{s}(r)-\omega_{s 0} \sim O\left(r^{2}\right)$ implying that, on the complex $-r$ plane, there is a conjugate pair of branch points at imaginary $r$ of order two (square-root singularities). The real $r$ axis remains on one of the two sheets and $\omega_{s}$ remains positive. If the branch points are permitted to converge on zero through variation of another parameter, the two sheets become disconnected except at the point $r=0$. The $r$ axis then has the choice of sheets that determines the sign of $\omega_{s}$ as $r$ crosses zero. Remaining on the same sheet (imagine that the branch points remain infinitesimally separated) results in $\omega_{s}$ reversing sign while $\omega_{s}$ keeps the same sign if $r$ jumps sheets. The latter results in the singular $\omega_{s}(r) \sim O(|r|)$ while the former results in the analytic $\omega_{s}(r) \sim O(r)$ as $r \rightarrow 0$. The latter case is a necessary condition that $r\left(\omega_{s}\right)$ is locally $C^{\infty}$. Although not shown here, it is also necessary that $U_{\mathrm{rf}}(r) \sim O\left(r^{4}\right)$ as $r \rightarrow 0$. For $r\left(\omega_{s}\right)$ to be globally $C^{\infty}$, it is sufficient that $\omega_{s}(r)$ is everywhere $C^{\infty}$ and that $d \omega_{s}(r) / d r$ is finite and bounded from below by a positive number.

From here on it is assumed that $r=r\left(\omega_{s}\right)$ is $C^{\infty}$ for real $\omega_{s}$. Due to the symmetry of the rf Hamiltonian $H(\phi, p)$ in the momentum variable $p$, we have the relation $\phi\left(-\omega_{s}, \theta\right)=\phi\left(\omega_{s}, \theta+\pi\right)$ implying $F_{\mu}\left(n,-\omega_{s}\right)=$ $(-1)^{\mu} F_{\mu}\left(n, \omega_{s}\right)$. This permits the two terms of Eq. (11) to be written as the two-sided Fourier transform

$\tilde{M}_{\mu}^{m n}(\tau)=\pi i \mu \int_{-\infty}^{\infty} d \omega_{s} \frac{\partial \Psi_{0}}{\partial \omega_{s}} F_{\mu}^{*}\left(m, \omega_{s}\right) F_{\mu}\left(n, \omega_{s}\right) e^{-i \mu \omega_{s} \tau}$

As a Fourier transform, we need to only show that $\Psi_{0}$ and the Fourier coefficients $F_{\mu}(n, J)$ are analytic functions of $r$ to evaluate the asymptotics of $\tilde{M}_{\mu}^{m n}(\tau)$ [4]. The $\Psi_{0}$ factor is simple since $\Psi_{0} \propto e^{-U_{\mathrm{rf}}(r) / \sigma^{2}}$ and $U_{\mathrm{rf}}(r)$ is assumed analytic. The Fourier coefficients $F_{\mu}$ are defined by the integrals

$$
F_{\mu}(n, J)=\frac{1}{2 \pi} \int_{0}^{2 \pi} d \theta e^{i(n \phi(J, \theta)-\mu \theta)}
$$

By construction, the action-angle variables $(J, \theta)$ for $C^{\infty}$ (single-particle) equations of motion have specific analytic properties. The equations of motion are $C^{\infty}$ if $U_{\mathrm{rf}}(\phi)$ is $C^{\infty}$, which is assumed in this paper. The function $\phi(J, \theta)$ is the $\phi$-coordinate solution of the equations of motion: the $\theta$ dependence specifies the time dependence while the $J$ dependence corresponds to the initial position in phase space at which the equations are integrated. Infinite differentiability with respect to both of these variables (for $J>0$ ) can be shown through properties of flows of vector fields defined on manifolds [5] (the theorem of the reference is applied to the vector field :H: defined on the phase space, where $: H: \Psi=\{\Psi, H\}$ and the braces are the Poisson brackets). The theorem also applies to the regime $r \leq 0$ by making use of the differentiability properties of $r=r(\phi, p=0)=\phi$ mentioned earlier.

Since $\phi(r, \theta)$ is $C^{\infty}$ in its $r$ dependence, the $r$ analytically continued function is necessarily regular in $r$ on and about the real axis. The integral Eq. (14) defining $F_{\mu}(n, J)$ is well behaved so $F_{\mu}(n, J=J(r))$ is necessarily regular in its $r$ dependence ([6], theorem 3.5.3).

This establishes that the integrand of Eq. (13) is analytic for real $r$ (and $\omega_{s}$ ). Since $\Psi_{0}(r) \rightarrow 0$ as $r \rightarrow \pm \infty$ as an exponential of a power and analytic conditions are met, $\tilde{M}_{\mu}^{m n}(\tau)$ defined by the Fourier transform Eq. (13) is necessarily bounded by a decaying exponential as $\tau \rightarrow \infty$ [4], ensuring that $M_{\mu}^{m n}(\Omega)$ is regular on and about the real- $\Omega$ axis. This is the result of this section.

\section{CONCLUSION}

An expression for the beam transfer function $G_{m n}(\Omega)$ in a basis of azimuthal harmonics that is the Fourier transform of a causal function $h_{m n}(\tau)$ was developed. This expression includes all orders of synchrotron harmonics and, when the causal function is precalculated, is an efficient way to numerically calculate dispersion relations. As a Fourier transform, it permits the evaluation of analytic properties of $G_{m n}$ through the large- $\tau$ asymptotics of $h_{m n}$. This was done numerically with an approximate form for $h_{m n}(\tau)$ and the $\phi^{4}$ rf potential. A condition ensuring that the terms $M_{\mu}^{m n}(\Omega)$ of $G_{m n}(\Omega)$ expressed in a series in synchrotron harmonics are regular on and about the real- $\Omega$ axis was also developed.

\section{ACKNOWLEDGMENTS}

The author thanks Jiunn-Ming Wang and Sam Krinsky for many helpful discussions.

\section{REFERENCES}

[1] S. Krinsky and J. M. Wang, Part. Accel. 17, 109 (1985).

[2] E. N. Shaposhnikova, Bunched Beam Transfer Matrices in Single and Double RF Systems, Tech. Rep. SL-94-19-RF, CERN - SL Division, Geneva, Switzerland (1994).

[3] A. N. Lebedev, At. Energ. 25(2), 100 (1968), [Sov. J. At. Energy, Vol. 25, p. 851-6 (1968)].

[4] G. E. Shilov, Elementary Functional Analysis (Dover Publications Inc., New York, 1974), sec. 5.2.

[5] R. L. Bishop and S. I. Goldberg, Tensor Analysis on Manifolds (Dover Publications Inc, New York, NY 10014, 1980), prop. 3.5.4.

[6] J. W. Dettman, Applied Complex Variables (Dover Publications, Inc., New York, 1965). 